\title{
Valoración del análisis de la pieza operatoria en el cáncer gástrico por el cirujano*
}

\author{
Drs. MARCO CERONI V. ${ }^{1}$, CARLOS GARCÍA C. ${ }^{1}$, RODRIGO VALLEJOS H. ${ }^{1}$, \\ JAIME ZAMARIN M. ${ }^{1}$, CARLOS BENAVIDES C. ${ }^{1}$, HÉCTOR CID B. ${ }^{1}$, PATRICIO RUBILAR O. ${ }^{1}$, \\ MARÍA ISABEL QUIJADA G. ${ }^{1}$, FRANCISCA SOLAR A. ${ }^{1}$, IGNACIO SOLAR A. ${ }^{1}$ \\ 1 Servicio y Departamento de Cirugía. Hospital Clínico San Borja Arriarán. Facultad de Medicina, \\ Campus Central, Universidad de Chile. \\ Santiago, Chile.
}

\begin{abstract}
Concordance between the surgical piece observation by the surgeon and final pathological report for gastric cancer

Background: The systematic dissection of the surgical piece, performed by the surgeon during surgical treatment of gastric cancer, gives information about borders and lymph node involvement. Aim: To determine the concordance between the findings of the surgeon during initial dissection and the final pathological report. Material and Methods: Prospective study of 48 patients aged $64 \pm 10$ years ( $74 \%$ males) subjected to curative surgery for gastric cancer. Patients were staged according to 2010 TNM classification. Stomach size from the lesser curvature, oral and caudal limits, macroscopic aspect, tumor diameter and lymph node involvement were determined by the surgeon observing the surgical piece. The concordance of this observation with the final pathological report was assessed. Results: Fifty nine percent of patients were subjected to a total gastrectomy and there was a mean of 30 lymph nodes excised. There was a good concordance between surgeon observation and final pathological report for tumor depth (Kappa $=0.64)$, macroscopic aspect $(\mathrm{Ka}-$ ppa $=0.69)$ and tumor size $(\mathrm{Lin}=0.84)$. There was a bad concordance for lymph node involvement (Kappa $=0.21$ ). The percentage of retraction of lesser curvature length was $24 \%, 30 \%$ for oral and $22 \%$ for caudal limits. Conclusions: There is a good concordance between surgeon observation and pathological report for macroscopic aspect, tumor size and depth but the concordance for lymph node involvement is bad.
\end{abstract}

Key words: Gastric cancer, surgical piece, surgeon.

\section{Resumen}

Introducción: La disección de la pieza operatoria (PO) en forma sistemática en la cirugía por cáncer gástrico (CG) permite al cirujano obtener información de los bordes, compromiso linfonodal y estandarizar. Objetivos: 1) Determinar la concordancia de los hallazgos de la PO disecada por el cirujano y el informe patológico final; 2) Determinar el porcentaje de retracción del estómago; 3) Mostrar una forma de registrar

*Recibido el 21 de octubre de 2010 y aceptado para publicación el 7 de diciembre de 2010.

Correspondencia: Dr. Marco Ceroni V.

El Vergel 2759 , Dpto. 504, Santiago, Chile.

marco.ceroni@gmail.com 
la PO en el CG. Material y Método: Estudio prospectivo de los pacientes operados con intención curativa desde septiembre de 2009 hasta mayo de 2010. Se etapificó con TNM de 2010. De la PO se registró el tamaño del estómago por curvatura menor, límite oral (LO), límite caudal (LC), profundidad, aspecto macroscópico, diámetro tumoral, grupos ganglionares comprometidos y cantidad de ganglios comprometidos. Se compararon estas variables con el informe patológico. El análisis de concordancia se realizó con el test de Kappa y el test de Lin. Resultados: Fueron estudiados 48 pacientes, 74\% hombres, la edad promedio fue 63,7 años (DE 9,8), 59,5\% fueron gastrectomías totales. El promedio de ganglios disecados fue 30,18 (DE 11,8). Se obtiene una buena concordancia para la profundidad del tumor (Kappa = 0,64), aspecto macroscópico (Kappa $=0,69)$ y tamaño tumoral $(\mathrm{Lin}=0,84)$. Y una mala concordancia para el compromiso ganglionar $($ Kappa $=$ 0,21). El porcentaje de retracción del largo por curvatura menor fue 24,3\%, del LO fue 30,1\%, del LC fue 22\%. Conclusión: La concordancia de la PO es buena en la profundidad, aspecto macroscópico del tumor y diámetro tumoral, pero es mala en el compromiso ganglionar. Se determina que la retracción del estómago es mayor al $24 \%$.

Palabras clave: Cáncer gástrico, pieza operatoria, gastrectomía total, gastrectomía subtotal.

\section{Introducción}

La cirugía curativa en el cáncer gástrico (CG) considera una serie de factores quirúrgicos de calidad bien conocidos entre los cirujanos oncólogos digestivos. El objetivo es lograr una cirugía sin tumor residual microscópico (R0), con una disección linfática extendida (D2) ${ }^{1}$, que considera un recuento mínimo de 16 ganglios linfáticos ${ }^{2}$, idealmente 25 ganglios linfáticos para una etapificación más precisa $^{3}$ y obtener márgenes macroscópicos negativos mayores de 5-6 cm, con confirmación microscópica ${ }^{4}$. El cumplimiento estricto de estos factores determina la optimización de los resultados oncológicos y mejora el pronóstico de los pacientes tratados ${ }^{5}$. Uno de los pasos indispensables para asegurar estos elementos es el análisis sistemático de la pieza operatoria (PO) por parte del cirujano, esto debido a que se puede cambiar la conducta quirúrgica en casos de márgenes quirúrgicos positivos o con límites inadecuados. Además, permite evaluar el compromiso ganglionar según la localización del tumor y comparar los resultados entre los distintos cirujanos y centros. Históricamente en nuestro hospital hemos realizado la disección de la PO en forma rutinaria, siguiendo los pasos de la escuela japonesa ${ }^{6}$, sin embargo, no se había estandarizado la forma de registro hasta ahora. Los objetivos del presente trabajo son: 1) Determinar la concordancia de los hallazgos de la PO disecada por el cirujano y el informe patológico final; 2) Determinar el porcentaje de retracción del estómago; 3) Mostrar una forma de registrar la PO en el CG.

\section{Material y Método}

Estudio prospectivo de los pacientes operados con CG con intención curativa, con disección linfática extendida desde septiembre de 2009 hasta mayo de 2010. La técnica consiste en la disección de la pieza operatoria por el cirujano con lupa, en una mesa anexa en el mismo pabellón posterior a la gastrectomía (Figura 1). Se recolectan los ganglios linfáticos por grupos ganglionares (Figura 2) según la clasificación japonesa, los cuales son separados en frascos rotulados, los que se envían a estudio

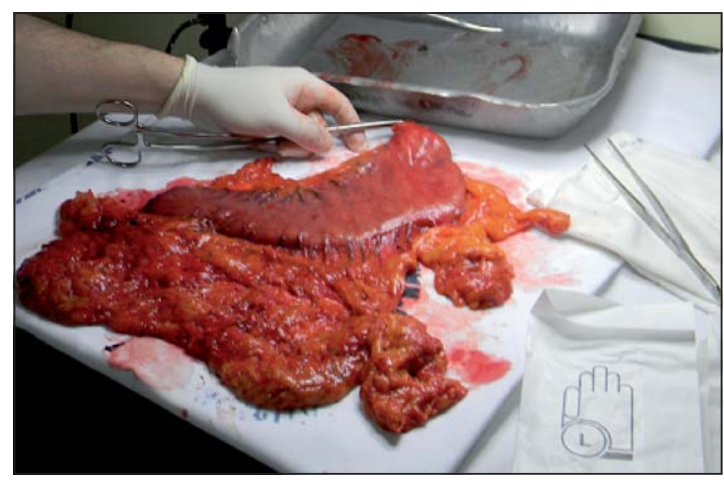

Figura 1. Pieza operatoria extendida en mesa anexa para su disección. Con frascos rotulados para la recolección de los ganglios linfáticos según grupos ganglionares.

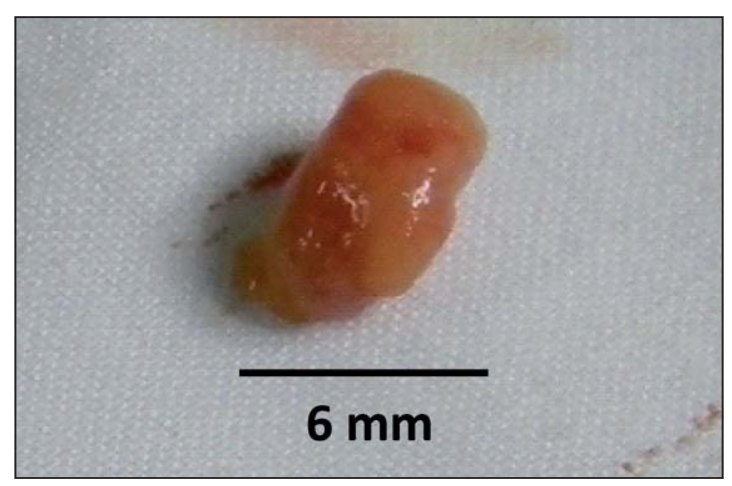

Figura 2. Ganglio linfático aislado. 


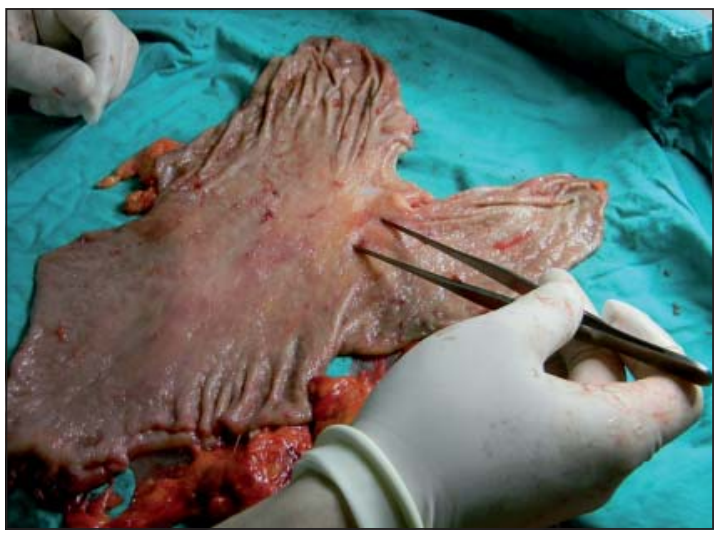

Figura 3: Estómago abierto por curvatura mayor, se observan dos lesiones incipientes subcardiales de $2 \mathrm{~cm}$ en espejo cerca de curvatura menor. diferido fijados en formalina al 10\%. A continuación se realiza la apertura del estómago por curvatura mayor (Figura 3), la medición de la pieza extendida y se registran las variables en una hoja estandarizada (Figuras 4 y 5). Se comparó el registro intraoperatorio de la $\mathrm{PO}$ con el informe definitivo del patólogo. Se realizó la etapificación con TNM de 2009 hasta diciembre de 2009; a partir de enero la nueva clasificación con TNM de 2010 y se registró en todos los pacientes la clasificación japonesa de 1995 por ser ésta más descriptiva que la de 1998. De la PO se registró el tamaño del estómago por curvatura menor, límite oral (LO), límite caudal (LC), profundidad, aspecto macroscópico, cantidad total de linfonodos disecados, tamaño del tumor, grupos ganglionares comprometidos y cantidad total de ganglios comprometidos por cáncer. Se compararon estas mismas

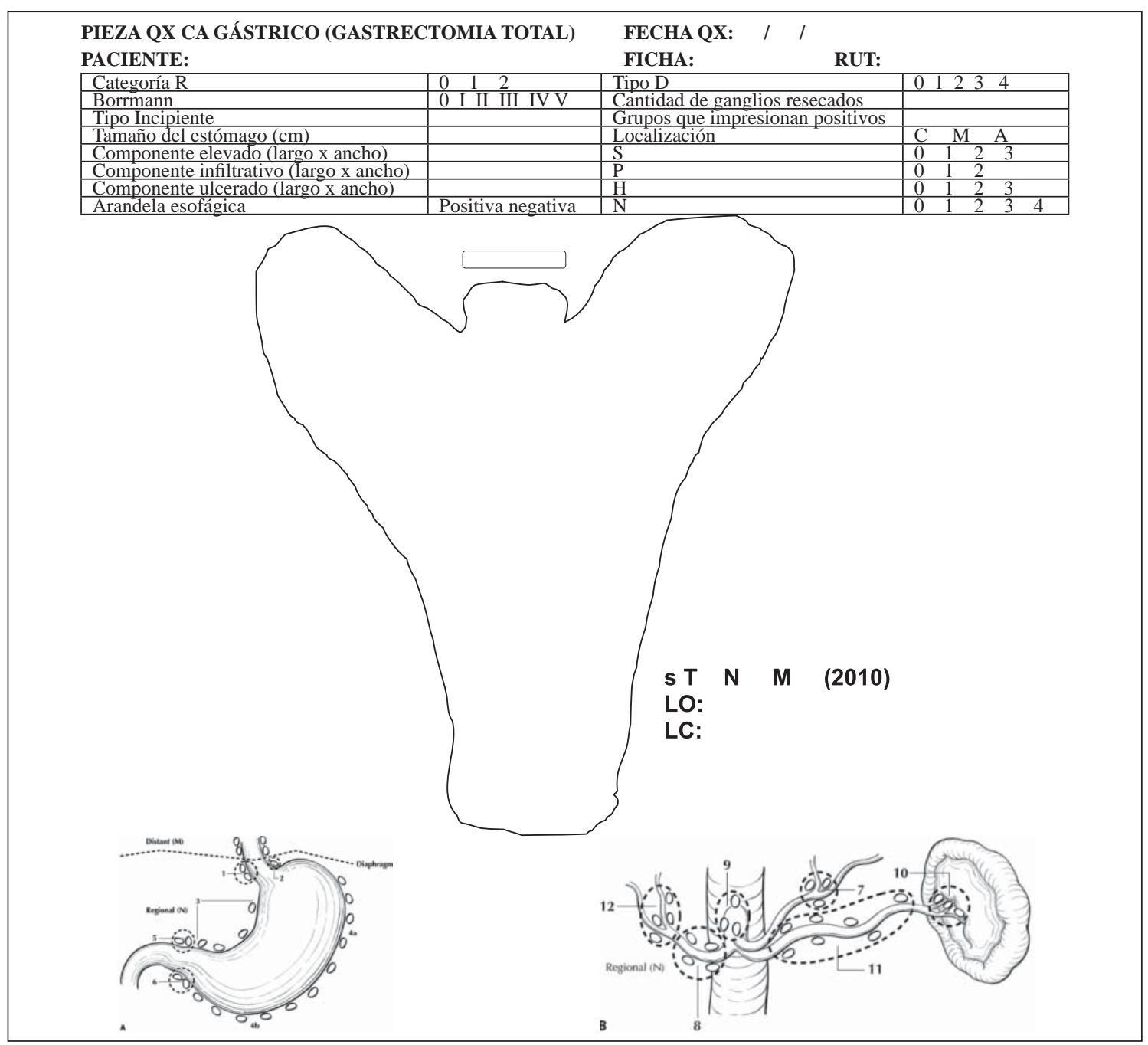

Figura 4a. Hoja de registro para gastrectomías totales en pacientes con cáncer gástrico. 
variables examinadas por el patólogo. El análisis de concordancia de las variables categóricas se realizó con el test de Kappa y para las continuas el coeficiente de correlación de concordancia de Lin. Se utilizó el test t de student para la comparación de los promedios. Se consideró una diferencia estadísticamente significativa con un valor $\mathrm{p}<0,05$. El análisis estadístico fue realizado con el programa estadístico Graph Pad Prism 5.

\section{Resultados}

La serie está constituida por 48 pacientes, 35 fueron de sexo masculino (74\%) y 13 pacientes de sexo femenino (26\%). La edad promedio fue de 63,7 años (DE 9,8; rango: 37 a 88 años). Un 59,5\% fueron gastrectomías totales. El promedio de ganglios disecados fue de 30,1 (DE 11,8; rango: 10 a 70). Se obtuvo una recolección de 25 o más ganglios en el $62,5 \%$ de los pacientes y menos de 16 ganglios en el $4,1 \%$ de los pacientes. Se obtiene una buena concordancia entre la evaluación del cirujano y el informe del patólogo para determinar la profundidad del tumor (Kappa = 0,64) (Tabla 1), el aspecto macroscópico (Kappa $=0,69)($ Tabla 2$)$ y para el tamaño del tumor $(\operatorname{Lin}=0,84)$. Se observa una mala concordancia para el compromiso ganglionar (Kappa $=0,21$ ) (Tabla 3). La estimación de la profundidad por el cirujano fue exacta en el 63,8\%, presentó una sobreestimación en el 25,5\% y una subestimación en el $12,7 \%$ de los casos. En el factor $\mathrm{N}$ de la nueva clasificación TNM de la AJCC, el cirujano realizó una estimación exacta en el 29,7\%, presentó una sobreestimación en el 38,2\% y una subestimación

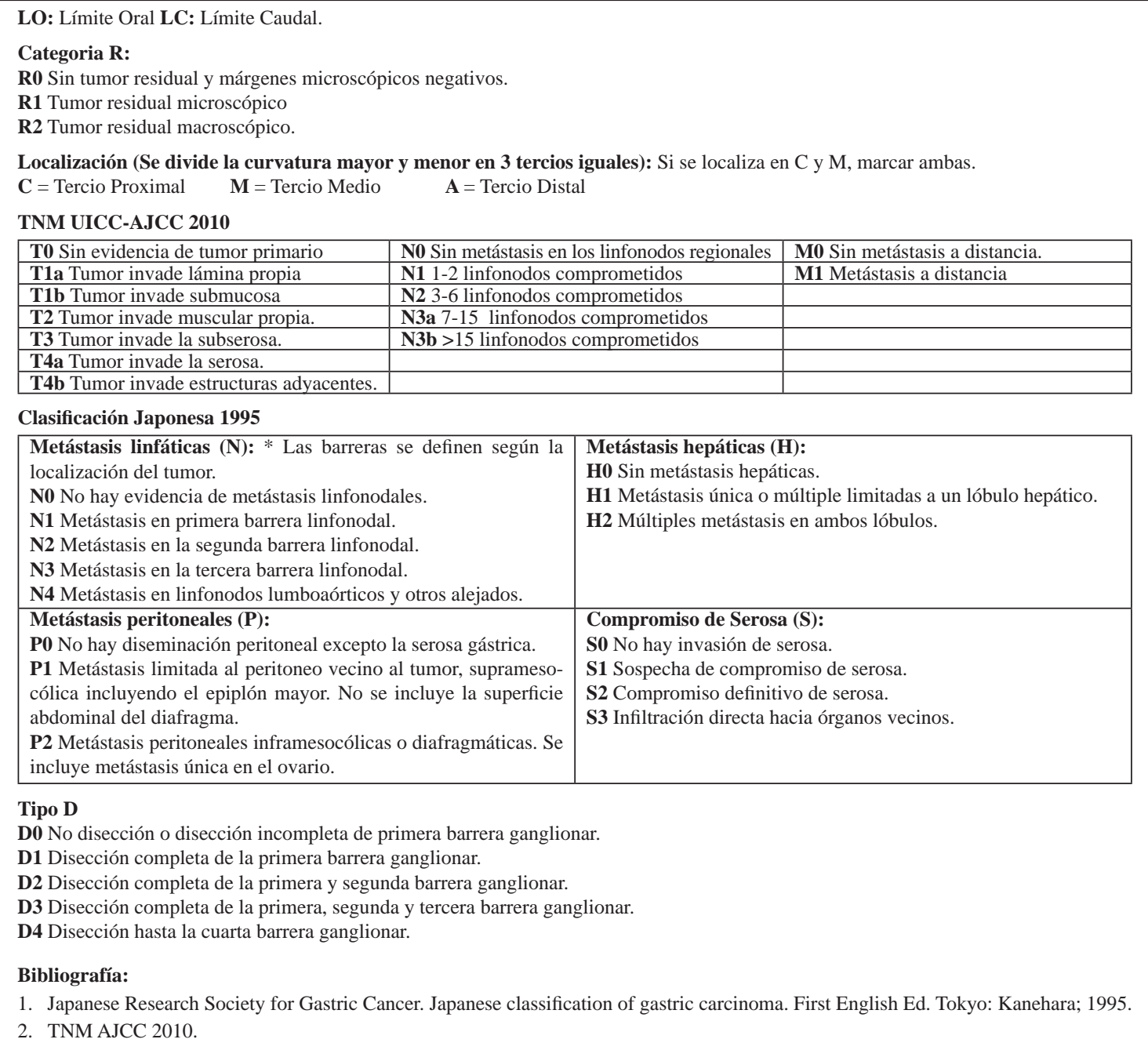

Figura 4b. Hoja de registro para gastrectomías totales en pacientes con cáncer gástrico (reverso). 
en el 31,9\% de los casos. La estimación exacta de los grupos ganglionares comprometidos tiene un promedio de 38,6\% (DE 36,3). El porcentaje de retracción del largo por curvatura menor globalmente fue de $24,3 \%$, del LO fue $30,1 \%$ y del LC fue $22 \%$. Al comparar las gastrectomías totales con las subto- tales se obtienen diferencias en el largo de la pieza operatoria $(\mathrm{p}=0,001)$, el LO ( $=0,0002)$ y LC $(\mathrm{p}<$ 0,0001). No se observan diferencias estadísticamente significativas en cuanto a la retracción de la PO, cambio del tamaño tumoral y cambio de tamaño de los márgenes quirúrgicos (Tabla 4).

Tabla 1. Evaluación de la profundidad del tumor presenta una buena concordancia $($ Kappa = 0,64)

\begin{tabular}{|c|c|c|c|c|c|c|c|}
\hline & \multicolumn{6}{|c|}{ Informe patológico } \\
\hline & & Incipiente & Muscular propia & Subseroso & Seroso & Extraseroso & \\
\hline \multirow{6}{*}{ 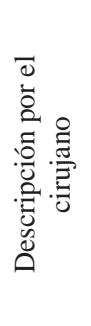 } & Incipiente & 6 & 2 & 0 & 1 & 0 & 9 \\
\hline & Muscular propia & 0 & 2 & 0 & 0 & 0 & 2 \\
\hline & Subseroso & 0 & 2 & 3 & 2 & 0 & 7 \\
\hline & Seroso & 0 & 1 & 8 & 18 & 1 & 28 \\
\hline & Extraseroso & 0 & 0 & 0 & 1 & 1 & 2 \\
\hline & & 6 & 7 & 11 & 22 & 2 & \\
\hline
\end{tabular}

Tabla 2. Evaluación del aspecto macroscópico presenta una buena concordancia $($ Kappa = 0,69)

\begin{tabular}{|c|c|c|c|c|c|c|c|c|c|c|}
\hline & & \multicolumn{9}{|c|}{ Informe patológico } \\
\hline & & B I & B II & B III & B IV & IIc & IIb & IIc+IIa & $\mathbf{I}$ & \\
\hline \multirow{11}{*}{ 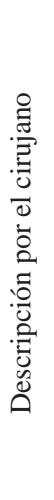 } & B I & 2 & 0 & 0 & 0 & 0 & 0 & 0 & 0 & 2 \\
\hline & B II & 1 & 2 & 7 & 0 & 0 & 0 & 0 & 0 & 10 \\
\hline & B III & 1 & 5 & 15 & 4 & 0 & 0 & 0 & 0 & 25 \\
\hline & B IV & 0 & 0 & 0 & 1 & 0 & 0 & 0 & 0 & 1 \\
\hline & B V & 0 & 0 & 0 & 1 & 0 & 0 & 0 & 0 & 1 \\
\hline & IIc & 0 & 0 & 0 & 0 & 2 & 0 & 0 & 0 & 2 \\
\hline & IIb & 0 & 0 & 0 & 0 & 2 & 0 & 0 & 0 & 2 \\
\hline & IIc+I & 0 & 0 & 0 & 0 & 0 & 1 & 0 & 0 & 1 \\
\hline & IIc+IIa & 0 & 0 & 0 & 0 & 0 & 1 & 1 & 0 & 2 \\
\hline & I & 0 & 0 & 0 & 0 & 0 & 0 & 0 & 1 & 1 \\
\hline & & 4 & 7 & 22 & 6 & 4 & 2 & 1 & 1 & \\
\hline
\end{tabular}

Tabla 3. Evaluación del factor $\mathbf{N}$ presenta una mala concordancia $($ Kappa $=\mathbf{0 , 2 1})$

\begin{tabular}{|c|c|c|c|c|c|c|c|}
\hline & & & & Inf & gico & & \\
\hline & & 0 & 1 & 2 & $3 \mathbf{A}$ & 3B & \\
\hline$\varpi$ & 0 & 6 & 3 & 0 & 0 & 0 & 9 \\
\hline & 1 & 3 & 0 & 3 & 5 & 0 & 11 \\
\hline 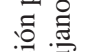 & 2 & 4 & 0 & 3 & 2 & 2 & 11 \\
\hline : & $3 A$ & 5 & 0 & 3 & 4 & 0 & 12 \\
\hline Uू & 3B & 0 & 1 & 0 & 2 & 1 & 4 \\
\hline & & 18 & 4 & 9 & 13 & 3 & \\
\hline
\end{tabular}


Tabla 4. Retracción de la PO, cambio de tamaño de los márgenes y del diámetro tumoral, separados según tipo de gastrectomía

\begin{tabular}{|c|c|c|c|c|}
\hline \multirow{2}{*}{ Curvatura menor } & Gastrectomía Total $(\mathrm{n}=25)$ & \multicolumn{2}{|c|}{ Gastrectomía Subtotal $(n=23)$} & \multirow[t]{2}{*}{$\mathbf{p}$} \\
\hline & & & & \\
\hline Largo Qx & $19,5 \mathrm{~cm} \quad \mathrm{DE} \quad 3,3$ (13 a 25) & 16,2 cm DE 3 & $(11,5$ a 23$)$ & 0,001 \\
\hline Largo AP & $14,7 \mathrm{~cm}$ DE 2,3 (10 a 20$)$ & $11,7 \mathrm{~cm} \mathrm{DE} \quad 1,7$ & $(8$ a 16$)$ & $<0,0001$ \\
\hline Retracción (cm) & 4,6 cm DE 2,8 (0 a 9) & $4,4 \mathrm{~cm} \quad \mathrm{DE} \quad 2,2$ & $(0,5$ a 10,5$)$ & \\
\hline Retracción (\%) & $22,7 \% \quad$ DE 12,3 (0 a 41,1) & $26,1 \% \quad$ DE 10,2 & $(4,3$ a 45,6$)$ & NS \\
\hline \multicolumn{5}{|l|}{ Límite oral } \\
\hline Largo Qx & 4,3 cm DE 2,4 (1 a 12) & DE 3,4 & $(2,2$ a 16$)$ & 0,0002 \\
\hline Largo AP & $2,4 \mathrm{~cm}$ DE $1,8(0,1$ a 7,5$)$ & $5,7 \mathrm{~cm} \quad \mathrm{DE} \quad 2,9$ & $(1,5$ a 13,5$)$ & $<0,0001$ \\
\hline Cambio tamaño (cm) & $-1,4 \mathrm{~cm}$ DE $2,1 \quad(-6,5$ a 2,5$)$ & $-1,84 \mathrm{~cm}$ DE 2 & $(-5,5$ a 1,5$)$ & \\
\hline Cambio tamaño (\%) & 37,9\% DE 34,5 (-50 a 90) & $23,1 \% \quad$ DE 24,8 & $(-27,2$ a 58,8$)$ & NS \\
\hline \multicolumn{5}{|l|}{ Límite caudal } \\
\hline Largo Qx & $12,1 \mathrm{~cm} \mathrm{DE} 5,5$ (1 a 20$)$ & $5,4 \mathrm{~cm} \quad \mathrm{DE} \quad 3,8$ & (1 a 13) & $<0,0001$ \\
\hline Largo AP & 7,8 cm DE 4,3 (0,1 a 14) & $4,6 \mathrm{~cm}$ DE 3 & $(0,5$ a 10$)$ & 0,0062 \\
\hline Cambio tamaño (cm) & $-3,6 \mathrm{~cm}$ DE $\quad 2,4 \quad(-9,5$ a 0,5$)$ & $-0,8 \mathrm{~cm} \quad \mathrm{DE} \quad 1,7$ & $(-4$ a 3$)$ & \\
\hline Cambio tamaño (\%) & $34,8 \% \quad$ DE $26,4(-7,1$ a 93,3$)$ & $10,2 \% \quad$ DE 35,6 & $(-80$ a 66,6$)$ & NS \\
\hline \multicolumn{5}{|l|}{ Diámetro del tumor } \\
\hline Largo Qx & 5,3 cm DE 2,6 (1 a 10) & $4,9 \mathrm{~cm} \quad \mathrm{DE} \quad 2,1$ & $(1,6$ a 9$)$ & NS \\
\hline Largo AP & 5,7 cm DE 2,7 (1,1 a 11) & $4,4 \mathrm{~cm} \quad \mathrm{DE} \quad 2,1$ & $(1 \mathrm{a} 8,5)$ & NS \\
\hline Cambio tamaño (cm) & 0,4 cm DE 1,4 (-1,8 a 5) & $-0,4 \mathrm{~cm} \quad \mathrm{DE} \quad 1,2$ & $(-3$ a 1,9$)$ & \\
\hline Cambio tamaño (\%) & $13,6 \% \quad$ DE $40 \quad$ (-60 a 150$)$ & $6,76 \%$ DE 32,8 & $(-60$ a 90$)$ & \\
\hline
\end{tabular}

\section{Discusión}

Los principales objetivos de los distintos sistemas de etapificación en el CG son predecir el pronóstico, decidir el plan de tratamiento, determinar el éxito de la cirugía y comparar los resultados entre los distintos centros 7 . La disección ganglionar por el cirujano permite recolectar una cantidad representativa de ganglios para su estudio patológico posterior. Nuestra serie obtuvo una recolección de 25 o más ganglios en el $62,5 \%$ de los pacientes y menos de 16 ganglios en el 4,1\% de los pacientes, lo que permite tener información adecuada del estatus ganglionar y poder definir la necesidad de quimioterapia adyuvante según el informe definitivo del patólogo.

Se pueden cometer errores en la etapificación, en los casos de disección de una cantidad insuficiente de ganglios se produce artificialmente un estadio $\mathrm{N}$ de la clasificación TNM de la AJCC-UICC menor al correcto ${ }^{7}$. A la inversa, si se diseca una gran cantidad de ganglios, aumenta la probabilidad de encontrar linfonodos comprometidos por cáncer y por lo tanto, aumentar el estadio $\mathrm{N}$, siendo que este grupo tenía inicialmente mejor pronóstico. Este efecto de la migración del estadio se conoce como fenómeno de Will Rogers ${ }^{8}$. Un elemento importante que corrige estos factores, que se considera desde hace algunos años, es el índice ganglionar, el cual corresponde al cociente entre los ganglios comprometidos por cáncer y el total de ganglios disecados de la $\mathrm{PO}^{9}$.

La estimación del compromiso ganglionar por cáncer de la PO por el cirujano es errática, tanto al evaluar la cantidad de ganglios comprometidos por cáncer como el compromiso según los grupos ganglionares definidos por la clasificación japonesa. Con una estimación exacta en nuestra serie que se acerca al 30\% y 40\%, respectivamente. Esto confirma lo establecido por otras series ${ }^{10-13}$.

El tamaño tumoral a pesar de tener una correlación excelente en nuestro trabajo, debe ser interpretado en forma cautelosa, aquí ocurren dos fenómenos, el primero, reconocido en la literatura ${ }^{4}$, es que el tamaño real del tumor es mayor al medido en la pieza fresca, debido a la extensión microscópica del tumor más allá de los márgenes evaluados macroscópicamente, el segundo, es la retracción del tumor debido a su fijación con formalina, como resultado se mantiene artificialmente el tamaño tumoral.

La experiencia desarrollada por nuestro grupo, demuestra que la evaluación sistemática de la PO en el cáncer gástrico permite lograr márgenes negativos. Se estima que el riesgo de tener márgenes positivos en las distintas series puede alcanzar un $7 \%{ }^{14,15}$. Otras medidas que ayudan a determinar los márgenes adecuados y el control local en forma qui- 
rúrgica, es la biopsia contemporánea y la reintervención precoz en los casos de compromiso tumoral.

La longitud recomendada para determinar un margen oncológicamente seguro varía entre los distintos estudios y el tipo de cirugía, esto debido a la existencia de un alargamiento y contracción natural distintos en cada parte del tracto digestivo. Para una adecuada definición de los márgenes oncológicamente seguros, se debe tener conocimiento del comportamiento del alargamiento por tracción del cirujano en la cirugía y la contractilidad en distintas condiciones del estómago. Se ha determinado que el alargamiento del estómago varía entre $15 \%$ y 22,6\%, según la tracción ejercida por el cirujano, cuya fuerza del brazo izquierdo se estima entre 500 y 1.000 grs. Una vez extraída la pieza presenta una contracción del 13,6\% y con formalina al 10\% a las 6-8 hrs de un $28,8 \%$. Con este conocimiento se ha determinado que el factor de corrección de las medidas del estómago son 1,41x a las 6-8 hrs de fijado y 1,43 x a las 12-24 hrs, respectivamente ${ }^{16}$.

El abordaje laparoscópico está validado en el tratamiento del cáncer gástrico incipiente ${ }^{17,18}$, sin embargo, la limitante principal es no poder identificar la lesión en forma adecuada en el acto quirúrgico, debido a no disponer del tacto del cirujano, por lo que en este punto la revisión de la PO es muy importante.

Uno de los tratamientos adyuvantes en el CG es la quimioterapia hipertérmica intraoperatoria con intención curativa, la cual se ha indicado en aquellos pacientes con compromiso seroso independiente de una citología positiva ${ }^{19,20}$. Esta terapia se considera debido a que el principal lugar de falla a distancia es la carcinomatosis peritoneal. La evaluación de la profundidad del tumor en la PO, ayuda a determinar la necesidad de este tratamiento, debido que presenta una buena concordancia al compararlo con el informe definitivo del patólogo.

En conclusión, la evaluación sistemática de la PO por el cirujano, es una herramienta fundamental en el tratamiento del CG, debido a que aporta información útil en el tratamiento quirúrgico en el control local de la enfermedad, permite obtener un muestreo ganglionar adecuado y evaluar la profundidad del tumor para determinar la necesidad de tratamiento adyuvante.

\section{Referencias}

1. Tanizawa Y, Terashima M. Lymph node dissection in the resection of gastric cancer: review of existing evidence. Gastric Cancer 2010;13:137-48.

2. Rausei S, Dionigi G, Boni L, Rovera F, Dionigi R. How does the 7th TNM edition fit in gastric cancer management? Ann Surg Oncol. 2010 sep 29 (online).

3. De Manzoni G, Verlato G, Roviello F. The new TNM classification of lymph node metastasis minimizes stage migration problems in gastric cancer patients. Br J Cancer 2002;87:17-4.

4. Bozzetti F, Bonfanti G, Bufalino R, Menotti V, Persano $\mathrm{S}$, Andreola S, et al. Adequacy of margins of resection in gastrectomy for cancer. Ann Surg. 1982;196:685-90.

5. Helyer L, O’Brien C, Coburn N, Swallow C. Surgeons' knowledge of quality indicators for gastric cancer surgery. Gastric Cancer 2007;10:205-14.

6. Japanese gastric cancer association. Japanese classification of gastric cancer -2th english edition-. Gastric Cancer 1998;1:10-24.

7. Ozgüç H, Sönmez Y, Yerci O. Metastatic/resected lymph nodes ratio-based classification in gastric cancer. Turk J Gastroenterol. 2008;19:2-7.

8. Bonder BE. Will Rogers and gastric carcinoma (letter). Arch Surg 1988;123:1023-4.

9. Yu W, Choi GS, Whang I, Suh S. Comparison of five systems for staging lymph node metastasis in gastric cancer. Br J Surg. 1997;84:1305-9.

10. Schmidt-Matthiesen A, Beyer P. Reliability of intraoperative macroscopic evaluation of lymph node metastasis and effectiveness of lymphadenectomy in stomach carcinoma. Abstract. Zentralbl Chir. 1996;121:121-5.

11. Park K, Chung S, McGuire L, Li A, Crofts T. Intraoperative assessment of lymph node involvement in gastric carcinoma. Ann R Coll Surg Engl. 1989;71:324-5.

12. Perng D, Jan C, Wang W, Chen L, Su Y, Liu G, et al. Computed tomography, endoscopic ultrasonography and intraoperative assessment in TN staging of gastric carcinoma. Abstract. J Formos Med Assoc. 1996;95:378-85.

13. Ziegler K, Sanft C, Zimmer T, Zeitz M, Felsenberg D, Stein $\mathrm{H}$, et al. Comparison of computed tomography, endosonography, and intraoperative assessment in TN staging of gastric carcinoma. Gut 1993;34:604-10.

14. Fujimoto S, Takahashi M, Mutou T, Kobayashi K, Toyosawa T, Ohkubo H. Clinicopathologic characteristics of gastric cancer patients with cancer infiltration at surgical margin at gastrectomy. Anticancer Res. 1997;17:689-94.

15. Venturelli F, Venturelli A, Cárcamo M, Cárcamo C, Jara C, Felmer O, Carrasco C. Gastrectomía subtotal en el cáncer gástrico antral mal diferenciado e indiferenciado. Rev Chil Cir. 2010;62:240-5.

16. Wang L, Shen J, Song X, Chen W, Pan T, Zhang W, et al. A study of the lenghening and contractily of the surgical margins in digestive tract cancer. Am J Surg. 2004;187:452-5.

17. Lee JH, Yom CK, Han HS. Comparison of long-term outcomes of laparoscopy-assisted and open distal gastrectomy for early gastric cancer. Surg Endosc. 2009;23:1681-3.

18. Huscher CG, Mingoli A, Sgarzini G, Sansonetti A, Di Paola M, Recher A, et al. Laparoscopic versus open subtotal gastrectomy for distal gastric cancer: five-year results of a randomized prospective trial. Ann Surg. 2005;241:232-7. 
M. CERONI V. y cols.

19. Fujimoto S, Takahashi M, Mutou T, Kobayashi K, Toyosawa T. Successful Intraperitoneal Hyperthermic Chemoperfusion for the Prevention of Postoperative Peritoneal Recurrence in Patients with Advanced Gastric Carcinoma. Cancer 1999;85:529-34.
20. Yan T, Black D, Sugarbaker P, Zhu J, Yonemura Y, Petrou G, et al. A Systematic Review and Meta-analysis of the Randomized Controlled Trials on Adjuvant Intraperitoneal Chemotherapy for Resectable Gastric Cancer. Ann Surg Oncol. 2007;14:2702-13. 